\title{
Highly ordered LB films of DHAP: a donor acceptor substituted polyene
}

\author{
M. Schmelzer and S. Roth \\ Max-Planck-Institut für Festköperforschung, Heisenbergstraße 1, 7000 Stuttgart 80 (Germany) \\ C.-P. Niesert and F. Effenberger \\ Institut für Organische Chemie, Universität Stuttgart, Pfaffenwaldring 55, 7000 Stuttgart 80 (Germany) \\ R. Li \\ 4 Physikalisches Institut, Universität Stuttgart, Pfaffenwaldring 57, 7000 Stuttgart 80 (Germany)
}

(Received January 20, 1993; accepted May 14, 1993)

\begin{abstract}
The donor acceptor substituted polyene 5-(4-dihexadecylaminophenyl)-2-methyl-2,4-pentadienal (DHAP) is-due to its amphiphilic nature - a promising candidate for the Langmuir-Blodgett (LB) technique. The molecule was studied as a monolayer on a pure water surface under argon atmosphere. Monolayers and multilayers were transferred onto solid support. The films showed a high degree of order in the monolayer as well as in thick multilayers. LB films were investigated with polarisation dependent UV/VIS absorption spectroscopy. The film spectra showed additional absorption peaks which were not present in the spectra of the molecules in solution. The relative peak intensities were highly dependent on the orientation of the electric field vector with respect to the substrate normal. To get a detailed picture of the arrangement of the films on the molecular level, we studied the polarisation dependence of the FTIR spectra in different experimental set-ups. The spectra showed a perpendicular orientation of the conjugated system with respect to the substrate, whereas the backbone of the saturated hydrocarbon chains showed a medium tilt angle of $20.0^{\circ}$ with respect to the substrate normal. X-ray small angle diffraction measurements were applied to investigate the thickness of the LB layers. From the position of the Bragg peaks, a thickness of $5.45 \mathrm{~nm}$ per bilayer can be calculated, in agreement with a bilayer model showing different molecular alignment of the molecules transferred during upstroke and downstroke.
\end{abstract}

\section{Introduction}

Following the trend of decreasing electronic device sizes, some years ago the idea of so-called molecular electronic devices was born (see for example ref. 1): information should be stored and perhaps even processed on a molecular level. Several "switching molecules" have been designed and synthesised. Usually the molecules are switched between the ground state and a metastable excited state by the absorption of light. The desired molecular properties may be tailored by chemical synthesis, but for many physical investigations the molecules have to be properly aligned (typical examples are second-order nonlinear optical effects); one way to achieve such an alignment is the LB technique [2].

With this in mind, several molecules were designed and tested as LB films. Some emphasised the amphiphilic character [3] necessary for film formation; others were designed to improve the switching process [4]. For our molecule, we have a donor acceptor substituted polyene, guaranteeing the function of a switching molecule. Long aliphatic chains and the aldehyde group take the amphiphilic character of potential LB candidates into account.

\section{LB film preparation}

In order to achieve LB film forming compounds with potential opto-electronic applications, we synthesised the donor bridge acceptor molecule DHAP, shown in Fig. 1, following the synthetic route reported earlier [5]. The dihexadecylaminophenyl part was selected because of its well known hydrophobic quality, including a high electron donating character [6]. Although only a weak electron acceptor, the hydrophilic aldehyde group was connected via a short unsaturated chain, promising good transferability of the LB films in monolayer and multilayer thickness onto solid support [7].

We used pure water purified in a Milli-Q-System (Millipore) as the subphase. The specific resistivity was $18 \mathrm{M} \Omega \mathrm{cm}$, and a total organic carbon content of less than $50 \mathrm{ppb}$ was achieved. The LB films were prepared 


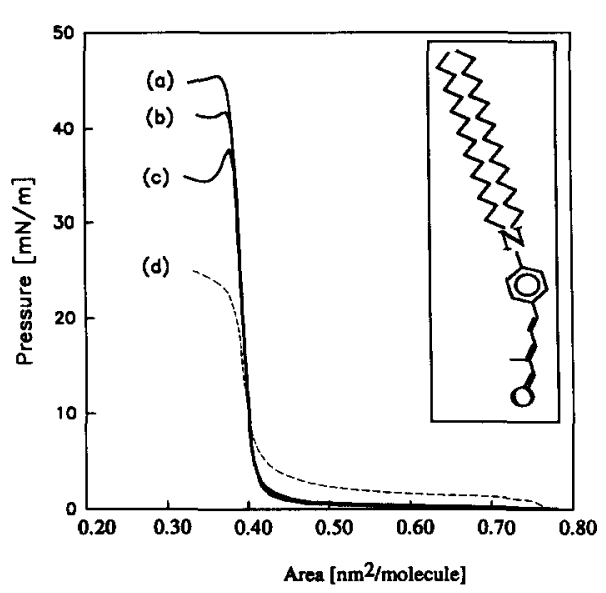

Fig. 1. Pressure area isotherms for several temperatures of the subphase: (a) $5.4{ }^{\circ} \mathrm{C}$, (b) $7.1^{\circ} \mathrm{C}$, (c) $8.8^{\circ} \mathrm{C}$, (d) $10.4^{\circ} \mathrm{C}$. The spectra were recorded with approximately $10^{17}$ molecules on the subphase. The inset shows a schematic representation of DHAP.

under an argon atmosphere by spreading a $1.5 \times 10^{-3} \mathrm{M}$ solution of DHAP in chloroform (Merck Uvasol quality). The purity of the solution was controlled via high performance liquid chromatography (HPLC). The molecule was seen to be degraded in solution within a few minutes when exposed simultaneously to light and atmosphere. In the HPLC chromatogram of the exposed molecules, two fractions were distinguishable due to a cis-trans isomerisation of the conjugated chain, so the solution was freshly prepared before spreading and the film balance (LAUDA FW1) was installed under yellow light conditions (clean room, class 1000) in a laminar flow box (class 100).

Figure 1 shows the pressure-area isotherms of the film on the pure water surface at different temperatures. At $5.4{ }^{\circ} \mathrm{C}$, film stabilities up to $45 \mathrm{mN} \mathrm{m}^{-1}$ can be achieved while compressing the film with $0.06 \mathrm{~nm}^{2}$ molecule ${ }^{-1} \min ^{-1}$. The area of the monolayer at the collapse point is $0.38 \mathrm{~nm}^{2}$ molecule ${ }^{-1}$, indicating a tight packing of the saturated hydrocarbon tails. At $7.1^{\circ} \mathrm{C}$ and constant pressure $\left(15 \mathrm{mN} \mathrm{m}^{-1}\right)$, the film was stable for several hours without detectable decrease of the area. When replacing the argon by normal atmosphere, the monolayer was degraded within $10 \mathrm{~min}$, showing an increasing area at constant pressure. Going to higher temperatures, the isotherms showed a liquid analogue phase, while collapse pressure and film stability, even at pressures $<15 \mathrm{mN} \mathrm{m}^{-1}$, decreased. For film transfer we used Suprasil glass slides as substrates for the UV/VIS absorption experiments. The glass was cleaned following the RCA procedure $[8,9]$ (boiling the substrates in a mixture of $\mathrm{NH}_{3}, \mathrm{H}_{2} \mathrm{O}_{2}$ and $\mathrm{H}_{2} \mathrm{O}$ (1:1:5) for $15 \mathrm{~min}$ ), rinsed with Milli-Q purified water, and dried under a stream of argon before use. The substrates were hydrophilic. If a hydrophobic substrate was desired, the cleaned glass slides were treated for 2 min with an ultrasonic bath containing hexamethyldisilazane. Then the glass was rinsed with methanol and pure water and dried under argon. For the FTIR absorption experiment in grazing incidence geometry we used a silver film $200 \mathrm{~nm}$ thick evaporated on an $\mathrm{Si}$ (111) surface precoated with $20 \mathrm{~nm}$ of Cr. For the FTIR transmission experiment we used a Si (111) single crystal. The crystal was RCA cleaned, etched with HF (30\%), rinsed with pure water, and dried with argon before use.

At $7.1{ }^{\circ} \mathrm{C}$ and $15 \mathrm{mN} \mathrm{m}^{-1}$ the film was transferred as a monolayer or multilayers onto the substrate. The transfer ratio was constant ( 0.4 during downstroke, 1.0 during upstroke) at dipping velocities of $24 \mathrm{~mm} \mathrm{~min}^{-1}$ for the downstroke and $3 \mathrm{~mm} \mathrm{~min} \mathrm{~m}^{-1}$ for the upstroke. The low transfer ratio of 0.4 for the downstroke can be explained as follows: if the hydrocarbon tails of the molecules are tilted at $66^{\circ}$ with respect to the substrate normal, the area per molecule is 2.5 times the area of upright standing molecules. Then an average orientation should be detected in polarisation-dependent FTIR absorption experiments, giving a medium tilt angle of the two orientations transferred during upstroke and downstroke. On the other hand, this model results in a bilayer thickness smaller than that of identically arranged upright or slightly tilted molecules.

\section{Characterisation of monolayers and multilayers}

\subsection{UV/VIS absorption}

Figure 2 shows the absorption spectrum of an LB film (20 layers on each side of the substrate) compared with the spectrum of the dissolved molecule. The main absorption shows a significant solvatochromic shift and appears, depending on the solvent, between $400 \mathrm{~nm}$ (in $n$-hexane solution) and $428 \mathrm{~nm}$ (in chloroform solution). This peak appears in the spectrum of the LB film

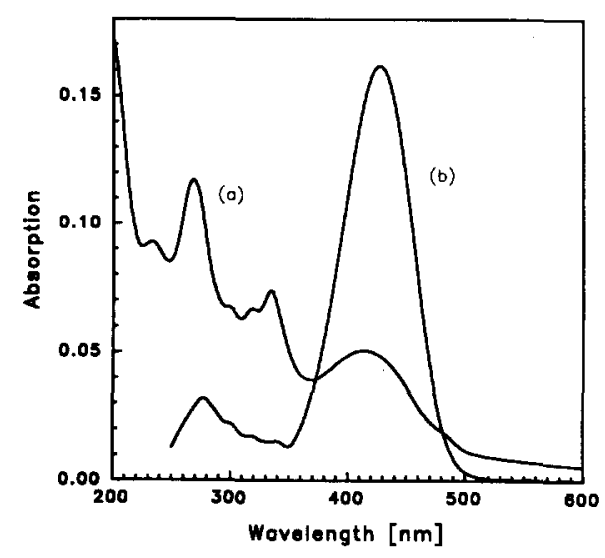

Fig. 2. UV/VIS absorption spectra of (a) the LB film and (b) the molecule (all trans) in chloroform solution. The spectra were recorded with a resolution of $2 \mathrm{~nm}$. 


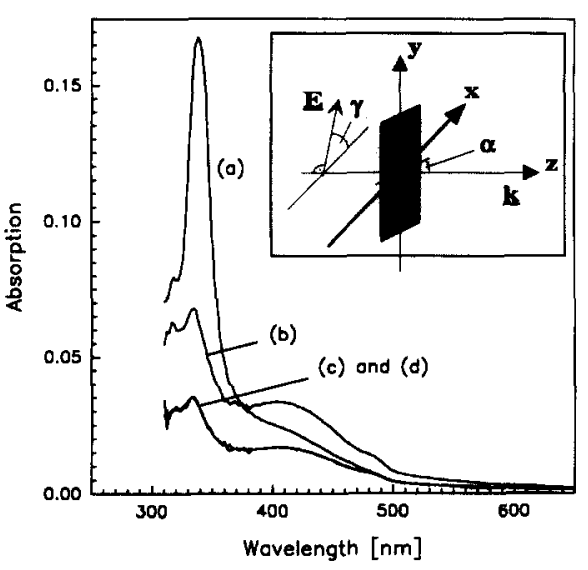

Fig. 3. Schematic representation of the experimental set-up with the polarisation-dependent UV/VIS absorption spectra. The geometry was (a) $\alpha=25^{\circ}, \gamma=0^{\circ}$; (b) $\alpha=25^{\circ}, \gamma=90^{\circ}$; (c) $\alpha=90^{\circ}, \gamma=90^{\circ}$; (d) $\alpha=90^{\circ}, \gamma=0^{\circ}$. All spectra were recorded with $2 \mathrm{~nm}$ resolution.

at $413 \mathrm{~nm}$. The second peak of the solution spectrum shows a solvatochromic shift and appears between $269 \mathrm{~nm}$ (in $n$-hexane solution) and $276 \mathrm{~nm}$ (in chloroform solution). The corresponding peak of the LB film spectrum appears at $268 \mathrm{~nm}$. Additional strong features at $334 \mathrm{~nm}$ can be detected in the spectrum of the LB film, which in the case of the solution appear only as weak modulations. These can be explained as follows: transitions between molecular levels, which are forbidden by symmetry selection rules for isolated molecules, can become allowed or the value of the corresponding dipole matrix elements for allowed transitions can be changed in the LB film due to the two-dimensional arrangement of the molecules in the LB matrix. The intensities of the absorption spectra show a linear dependence on the number of transferred LB layers, indicating a homogeneous transfer of the multilayers. No further interaction of the optically active part of DHAP perpendicular to the plane of the LB film can be detected.

To examine the ordering pattern of the LB films, we have carried out polarisation dependent UV/VIS absorption experiments. Figure 3 shows a schematic representation of the experimental set-up and a selection of the spectra of an LB film (ten layers on each side of the Suprasil). The spectra show a very strong dependence of the relative peak intensities on the orientation of the electric field vector with respect to the substrate normal. While in case (b) the peak at $413 \mathrm{~nm}$ is easily detectable, it decreases in intensity when the electric field vector is rotated out of the substrate surface (a). The peak at $334 \mathrm{~nm}$ shows a marked increase in intensity during the same rotation. This clearly indicates a high degree of order of the optically active part of DHAP, which is the conjugated part of the molecule. The spectra show no polarisation dependence when the elec- tric field vector is rotated around the $z$-axis while the substrate is kept in the $x-y$ plane. Therefore, no preferred orientation of the LB films with respect to the dipping direction can be detected. The stronger intensity in (b) than in (c) is due simply to a difference in the illuminated area, which increases with $1 / \sin \alpha$. The same strong polarisation dependence of the UV/VIS absorption spectra shown in Fig. 3 was recorded for thicknesses of $20 \mathrm{LB}$ layers down to a single monolayer transferred on each side of a hydrophilic Suprasil glass slide. All spectra were recorded on a commercial UV/ VIS spectrometer (Perkin-Elmer, Lambda 2) under normal atmosphere and the spectra were reproducible provided that the films were not exposed to daylight under atmosphere. If illuminated for more than $10 \mathrm{~min}$, the samples would degrade.

\subsection{FTIR spectroscopy}

In order to confirm the arrangement of the LB films of DHAP and to get a detailed picture of the molecular arrangement, we have carried out polarisation-dependent FTIR experiments. Transmission FTIR and grazing incidence FTIR were recorded on a commercial spectrometer (Bruker $113 \mathrm{v}$ ), using a wire-grid polariser ( $200 \mathrm{~nm} \mathrm{Au}$ on KRS5). All spectra were obtained on freshly prepared films, directly transferred into the vacuum chamber of the spectrometer.

Figure 4 shows a schematic representation of the experimental set-up and the spectra of the LB films. Table 1 summarises the correlation between the detected peaks and the corresponding molecular vibra-

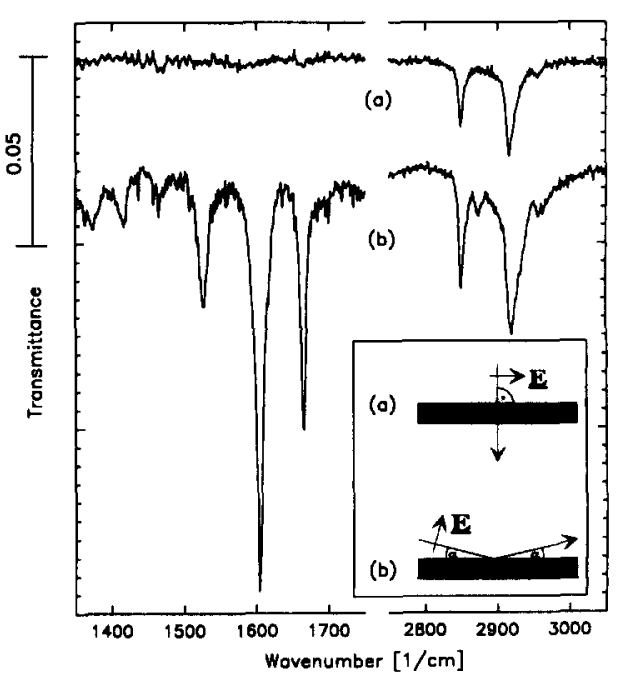

Fig. 4. Schematic representation of the experimental set-up for the polarisation-dependent FTIR experiments. For clarity, the spectrum corresponding to the grazing incidence geometry (b) has been shifted with respect to the spectrum corresponding to the transmission experiment (a). The spectra were recorded with a resolution of $2 \mathrm{~cm}^{-1}$. The data shown are measured data without further manipulation (smooth, background subtraction, etc.) 
TABLE 1. Correlation of the FTIR features and the molecular vibrations with the relative intensities in the two different geometries: $w=$ weak, $s=$ strong, $v s=$ very strong, $s h=$ shoulder, $\delta=$ deformation, $v=$ stretching mode

\begin{tabular}{|c|c|c|}
\hline $\begin{array}{l}\text { Transmission geometry } \\
\text { wave number }\left(\mathrm{cm}^{-1}\right) \text {, } \\
\text { intensity }\end{array}$ & $\begin{array}{l}\text { Grazing incidence } \\
\text { geometry } \\
\text { wave number }\left(\mathrm{cm}^{-1}\right) \text {, } \\
\text { intensity }\end{array}$ & $\begin{array}{l}\text { Molecular } \\
\text { vibration }\end{array}$ \\
\hline - & $1375, \mathrm{w}$ & $\delta\left(\mathrm{CH}_{3}\right)$ \\
\hline- & $1418, w$ & $v(\mathrm{C}=\mathrm{C})$ \\
\hline- & $1465, w$ & $\delta\left(\mathrm{CH}_{2}, \mathrm{CH}_{3}\right)$ \\
\hline - & $1527, \mathrm{~s}$ & $v(\mathrm{C}=\mathrm{C})$ \\
\hline - & 1604 , vs & $v(\mathrm{C}=\mathrm{C})$ \\
\hline- & $1665, \mathrm{~s}$ & $v(\mathrm{C}=\mathrm{O})$ \\
\hline $2850, \mathrm{~s}$ & $2851, \mathrm{~s}$ & $v_{s}\left(\mathrm{CH}_{2}\right)$ \\
\hline - & $2874, w$ & $v_{s}\left(\mathrm{CH}_{3}\right)$ \\
\hline $2917, \mathrm{~s}$ & $2919, \mathrm{~s}$ & $v_{\mathrm{a}}\left(\mathrm{CH}_{2}\right)$ \\
\hline- & 2930, sh & $v_{\mathrm{s}}\left(\mathrm{CH}_{3}\right)$ \\
\hline 2956, w & $2960, w$ & $v_{\mathrm{a}}\left(\mathrm{CH}_{3}\right)$ \\
\hline
\end{tabular}

tions. The features are in excellent agreement with earlier reported data on similar molecules [10-12]. Spectrum (a) shows the result of the transmission experiment ( 14 layers on each side of the substrate). In this case the electric field vector is in the plane of the substrate surface. The peaks in the region between $2750 \mathrm{~cm}^{-1}$ and $3050 \mathrm{~cm}^{-1}$, corresponding to the saturated hydrocarbon chains, dominate the spectrum in this geometry. No peak appears in the region between $1350 \mathrm{~cm}^{-1}$ and $1750 \mathrm{~cm}^{-1}$, where the vibrations due to the aldehyde group and the conjugated chain are expected. Spectrum (b) shows the result of the grazing incidence experiment (14 layers, $\alpha=6^{\circ}$ ) with the electric field vector perpendicular to the plane of the surface. The spectrum shows very intense peaks in the region between $1350 \mathrm{~cm}^{-1}$ and $1750 \mathrm{~cm}^{-1}$, which dominate the peaks in the region between $2750 \mathrm{~cm}^{-1}$ and $3050 \mathrm{~cm}^{-1}$.

Comparing the spectra in the low wavenumber region, it can be deduced directly that the backbone of the conjugated system is oriented perpendicular to the plane of the substrate surface. The vibrations can be excited (case (b)) only when the electric field vector has a component parallel to the symmetry axis of the oscillation which is the backbone of the conjugated system. We have evaluated the peak intensities of the saturated hydrocarbon stretch vibrations in our geometries (a) and (b) following a method proposed by Chollet et al. [1-3] and developed by Umemura et al. [14], with the use of a formalism developed by Hansen [15]. The average tilt angle of the backbone of the saturated hydrocarbon chains was calculated to be $20.0^{\circ}$ with respect to the substrate normal. Taking into account the length of the constituent units of DHAP, the bilayer thickness of $6.1 \mathrm{~nm}$ is expected for the model of homogeneously oriented films. The above-mentioned

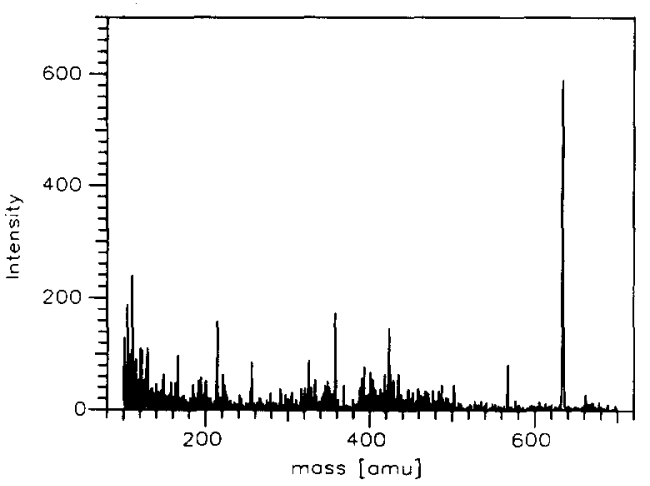

Fig. 5. Rest gas spectrum of the UHV chamber while the LB film was at $50^{\circ} \mathrm{C}$. The main peak at $635 \mathrm{amu}$ correlates with the molecule DHAP.

model of alternating upright and tilted arrangements results in a thickness of $5.2 \mathrm{~nm}$ per bilayer. The X-ray measurements reported below reveal that the latter model is correct.

FTIR experiments on a single monolayer transferred in a single upstroke onto an evaporated silver film were recorded in the grazing incidence geometry installed in a further spectrometer in a UHV chamber. The detected features are in excellent agreement with the data shown in Fig. 4(b). In particular, the peak at $1665 \mathrm{~cm}^{-1}$ corresponding to the vibration of the aldehyde part, which in this case is connected to the silver film, appears in the monolayer spectrum at the same wave number as in the multilayer spectrum. It can be deduced that there is only a weak coupling of the first monolayer to the substrate via Van der Waals interaction. This is compatible with the fact that the LB film completely desorbs upon heating of the substrate to $70^{\circ} \mathrm{C}$; this can be detected by monitoring the rest gas spectrum of the UHV chamber with a quadruple mass spectrometer while the substrate temperature is increased. Figure 5 shows a rest gas spectrum of the UHV chamber containing an elevenlayer-thick LB film at $50{ }^{\circ} \mathrm{C}$. The rest gas spectra further indicate that the molecules were transferred as intact molecules, and were not degraded during the LB preparation. The main peak is at a mass of $635 \mathrm{amu}$, which is in excellent agreement with the mass of $636 \mathrm{amu}$ calculated by simply adding the atomic masses.

\section{3. $X$-ray measurements}

Figure 6 shows the results of small angle X-ray diffraction measurements of ten monolayers of DHAP on $\mathrm{Si}$ (111). The data shown are the results as measured with a conventional diffractometer (Siemens D 500, $0.154 \mathrm{~nm}$ ) without further manipulation. Since there are no strong scatterers (such as heavy metals) in our molecule, only fairly weak features are seen. At $1.62^{\circ}$ we observe a pronounced Bragg peak. From the position of this peak we deduce a bilayer thickness of 


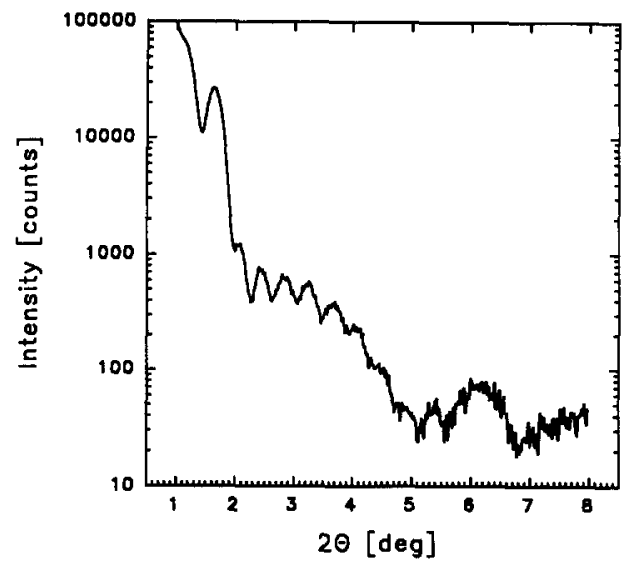

Fig. 6. X-ray small angle diffraction measurements of ten monolayers of DHAP on $\mathrm{Si}(111)$. The Bragg peak at $1.62^{\circ}$ indicates a bilayer thickness of $5.45 \mathrm{~nm}$.

$5.45 \mathrm{~nm}$. The modulations between $2^{\circ}$ and $6^{\circ}$ are due to interferences from the total LB film thickness. They are superimposed on the Bragg peaks of higher order. When the LB films of DHAP are illuminated with the $\mathrm{X}$-rays for several hours, the samples degrade and the Bragg peak at $1.62^{\circ}$ completely disappears. Therefore, the X-ray measurements were performed with medium angular resolution and high scan speed to avoid degradation.

\section{Conclusions}

We have demonstrated that it is possible to form LB films of DHAP, which is a potential candidate for optical and molecular electronic applications. The molecules build stable LB monolayers on the water surface; the intact molecules were transferred as monolayers and multilayers onto solid support. In polarisation-dependent UV/VIS absorption experiments and polarisation-dependent FTIR spectroscopy, the films showed a high degree of molecular alignment. The results clearly indicate a perpendicular orientation of the optically active part of the molecule, which is the conjugated system, with respect to the substrate surface. The saturated hydrocarbon chains which act as isolating layers between the active parts showed a medium tilt angle of $20^{\circ}$ with respect to the surface normal (Fig. 7). This molecular alignment represents an improvement over flat lying optically active parts [6], and makes the molecules good candidates for wave guiding experiments. Taking the results of X-ray experiments into account, a model of the bilayer was established, showing different orientations of the molecules for upstroke and downstroke transfer. In LB heterostructures containing DHAP a vectorial electron transfer is ex-

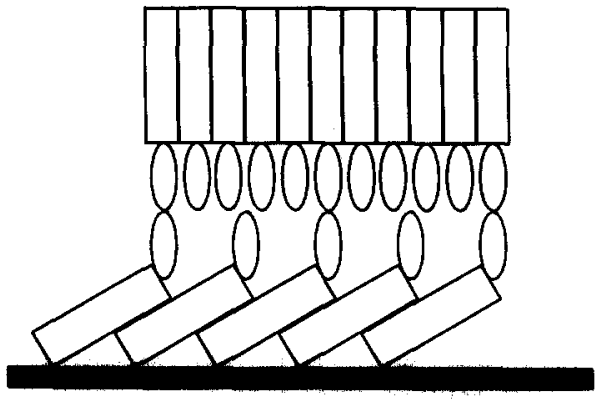

Fig. 7. The model of the bilayer shows the different orientations of the molecules transferred during upstroke and downstroke. The calculated bilayer thickness of $5.2 \mathrm{~nm}$ is in good agreement with the experimental results $(5.45 \mathrm{~nm})$. The boxes represent the saturated hydrocarbon chains, the ellipses represent the conjugated part of the molecule.

pected, resulting in a macroscopic detectable current in sandwich structures, when switching the molecules from HOMO to LUMO. Further investigations to determine non-linear optical effects are in progress, using the linear Pockels effects in the SPR set-up reported earlier [10].

\section{Acknowledgments}

We wish to thank the Deutsche Forschungsgemeinschaft under Sonderforschungsbereich 329 (Molekulare Elektronik) for financial support and P. Langjahr and $\mathrm{N}$. Karl (University of Stuttgart) for their help in the $\mathrm{X}$-ray measurements.

\section{References}

1 F. L. Carter, Molecular Electronic Devices I, Dekker, New York, 1982.

2 G. L. Gaines, Jr., Insoluble Monolayers at Liquid Gas Interfaces, Wiley-Interscience, New York, 1966.

3 S. Hagen, H. Schier, S. Roth and M. Hanack, in H. Kuzmany, M. Mehring and S. Roth (eds.), Electronic Properties of Conjugated Polymers, Kirchberg III, Vol. 91, Springer, Heidelberg, 1989, p. 398.

4 C. Bubeck, F. Effenberger, L. Häußling, D. Neher, C.-P. Niesert and H. Ringsdorf, Adv. Mater., 4 (1992) 413.

5 C.-P. Niesert, Ph.D. Thesis, Universität Stuttgart, 1992.

6 D. Lupo, W. Prass, U. Scheunemann, A. Laschewsky, H. Ringsdorf and I. Ledoux, J. Opt. Soc. Am., SB (1983), 300.

7 T. Wagner, S. Hagen, S. Roth, S. Akari and K. Dransfeld, Thin Solid Films, 210/211 (1992) 652.

8 W. Kern, D. A. Poutinen, RCA Rev., 6 (1970) 187.

9 W. Kern, RCA Engineer, 28 (1983) 99.

10 J. F. Rabolt, F. C. Burns, N. E. Schlotter and J. D. Swalen, J. Chem. Phys., 78 (1983) 946.

11 T. Wagner, Ph.D. Thesis, Universität Karlsruhe, 1992.

12 H. Schier, Ph.D. Thesis, Universität Stuttgart, 1992.

13 P.-A. Chollet, J. Messier and C. J. Rosilio, J. Chem. Phys., 64(1976) 1042.

14 J. Umemura, T. Kamata, T. Kawai and T. Takenaka, J. Phys. Chem., 94 (1990) 62.

15 W. N. Hansen, J. Opt. Soc. Am., 58 (1968) 380. 